\title{
Structure of Space Fabric
}

\section{Pramod Kumar Agrawal}

Universal Theory Research Centre, Jawahar Lal Nehru Marg, Jaipur, Rajasthan, India

Correspondence to: Pramod Kumar Agrawal, agrawalkpramod@gmail.com, pramod@universaltheoryonline.com Keywords: Space Fabric, Aether, Dark Matter, Spacetime, Emptiness of Space

Received: October 31, $2021 \quad$ Accepted: December 5, $2021 \quad$ Published: December 8, 2021

Copyright $\odot 2021$ by author(s) and Scientific Research Publishing Inc.

This work is licensed under the Creative Commons Attribution International License (CC BY 4.0).

http://creativecommons.org/licenses/by/4.0/

\section{Open Access}

\section{ABSTRACT}

This paper first proposes that a physical entity such as an electron and a cosmological entity such as a photon belong to two different levels of the Universe. When declaring that space is a vacuum, we are referring to the physical vacuum, and not the cosmological vacuum. The paper propounds that the space we observe is a fabric of cosmological substances. We cannot presume that something fills the emptiness of space. This paper posits that the fabric of cosmological substances creates this emptiness to facilitate physical activities such as electromagnetic waves and gravitational pull. Second, the space fabric is a spatial cosmological phenomenon, whereas time is a physical phenomenon operated by a physical entity. The spacetime continuum is created by physical time acquiring the cosmological space fabric. The third part of this paper explains the structure and ingredients of the space fabric. The structure itself explains several cosmological phenomena, including electromagnetic waves, gravitation, light, magnetism, dark matter, and dark energy. The philosophical correctness of the paper is validated through the unification of multidisciplinary aspects, and its scientific correctness is validated by its logical consistency with the results of well-known experiments.

\section{INTRODUCTION}

\subsection{Short History of the Aether}

An electromagnetic wave needs a medium to propagate through open space. An unknown substance named the aether is believed to fill empty space as a medium. Aristotle proposed that the aether is the fifth basic element, along with air, fire, water, and earth. In his seminal work "Principia", published in 1686, Newton described the aether as the medium of gravity. In his following work, "Optiks," published in 1704, he described the aether as the medium of electromagnetic waves. However, the Michelson-Morley experiment cast doubt on the aether in 1887. This suspicion remained until 1915 when Albert Einstein introduced his theory of relativity, and the term "fabric of spacetime" was popularized. Albert Einstein stated in 1920 [1] that "... space without aether is unthinkable; for in such space there not only would be no propa- 
gation of light, but also no possibility of existence for standards of space and time (measuring-rods and clocks), nor therefore any spacetime intervals in the physical sense. But this aether may not be thought of as endowed with the quality characteristic of ponderable media, as consisting of parts which may be tracked through time. The idea of motion may not be applied to it...”. The presence of the aether was thus established.

\subsection{Historical Understanding of the Structure of Aether or Space Fabric}

In 1644, René Descartes proposed an all-pervading aether with mechanical properties. He assumed that "the Aether particles are continually in motion." He inferred that "This motion constitutes closed chains creating vortices." Some philosophers presume that the aether is a continuous, flat, and single fundamental substance. However, this view contradicts General Relativity, which assumes that the geometric structure of spacetime is contingent and determines gravity; furthermore, a single substance of infinite size is unlikely. Some philosophers believe that the aether is made of small granules but do not describe what fills the gap between the granules. Nerlich argued [2] that "It may well be possible to use the symmetries to fill in the gaps." Other philosophers believe that these granules overlap to fill the gaps. Cockaday stated that "we must rather accept the view that all space is filled with a gaseous substance." According to Thomson and Bourassa [3], a quantum aether is "simultaneously solid-fluid-gaseous", which looks like tubes, called "spin positions." These spin positions are cardioidal structures curved by [a] "spherical distributed frequency." They agreed with Mach that "Aether acts on matter, and matter in turn acts upon Aether." Peter Forrest [4] suggested some potential structures of aether. Nafousi stated [5] that "...Empty space is not empty but composed of space particles $(S P) \ldots$... Among the prevailing theories of the aether's structure, a satisfactory theory is still lacking [6].

\subsection{Modern Understanding of the Structure of Aether or Space Fabric}

Throughout the Universe, underlying background energy exists. This energy, called vacuum energy, is a special case of zero-point energy that relates to the quantum vacuum and is the basis of the space fabric or aether. The existence of vacuum energy has been demonstrated by the Casimir effect, spontaneous emission, and the Lamb shift. The author suggests that this vacuum energy is merely a different cosmological substance. This paper provides several philosophical and scientific arguments for understanding this cosmological substance.

\subsection{Achievements of the Article}

By adopting a philosophical perspective, this article aims for a logical and simplified description of space. Some important achievements of the article are listed below.

1) As the physical and cosmological worlds exist on different levels, we cannot presume that luminiferous aether (a cosmological substance) will follow the laws of physical science. The aether, as a means, enables physical activations but cannot physically perform those activations.

2) The space fabric (aether) does not fill empty space. On the contrary, emptiness itself is composed of the space fabric. If there is no space fabric, there can be no emptiness, and space cannot exist.

3) The space fabric (aether) and spacetime are two different entities. The space fabric is a cosmological phenomenon pervading the Universe. Whereas time is the activation of a physical entity and remains active until the space fabric is physically activated in the form of electromagnetic waves or gravitational pull. Time cannot exist without the space fabric, but the space fabric can exist without time.

4) The paper explains the structure and ingredients of the space fabric and explains how this structure resolves different mysteries of quantum physics.

5) The fabric structure is uniform but entirely complies with the general theory of relativity. The space fabric comprises an infinite number of primary cosmological units, each with its individual cosmological arrangement and curvature, which are knotted into a continuous fabric with cosmological bindings. 
6) The proposed model of space fabric can explain the activation of electromagnetic waves, gravitation, dark energy, dark matter, and other mysteries of the Universe.

\section{METHODOLOGY}

The methodology is based on the fact that unitary logic is applicable to all entities of the Universe. As explained above, there are five known levels of the Universe [7, 8]. In order of the lower (earlier) to upper (later) levels, these levels are defined as the following: cosmological level (energy fields), physical level (atoms), biological level (plants), psychological level (animals), and intellectual level (humans) [9]. The logic of the unitarity suggests that every two successive levels have a parallel relationship, which can be used to understand the space fabric.

1) In the first part (see Section 3.1 of the paper), we will discuss the presence of the cosmological space fabric based on the other levels of the Universe.

2) In the second part (see Section 3.2 of the paper), we will discuss the differences between the physi$\mathrm{cal}$ and cosmological entities. For example, the psychological phenomenon is a means of activation of the intellectual entity. The biological phenomenon is a means of activation of the psychological entity, and the physical phenomenon is a means of activation of the biological entity. Hence, we can easily infer that the cosmological phenomenon is a means of activation of the physical entity. Thus, we can conclude that the cosmological entities are physically activated when physical time activates them.

3) In the third part (see Section 3.3 of the paper), we will discuss the panoramic view of the Universe, which explains the creation and scope of the space fabric.

4) In the fourth part (see Section 3.4 of the paper), we will discuss the emptiness of space.

5) In the fifth part (see Section 3.5 of the paper), we will discuss the difference between the space fabric and spacetime.

6) In the sixth part (see Section 4 of the paper), we will discuss the structure of the primary unit of the fabric.

7) In the seventh part (see Section 4.5 of the paper), we will discuss the evolution of the energy fields from the primary unit.

8) In the eighth part (see Section 5 of the paper), we will discuss the applicability of these four fields in different cosmological mysteries.

\section{DISCUSSION AND PROOFS}

\subsection{Unification of Multidisciplinary Aspects}

The methodology is based on the idea that nature behaves equanimously with all entities. A proven fact can be used in other situations, using the same logic with parallel aspects. It is a known fact that an intellectual time (involvement of brain) activates (through knowing, thinking, and explaining) the space fabric (mind) composed of psychological substances (emotions) [10-14]. Psychological time activates (through desire, excitement, and fear) the space fabric (temperament) composed of biological substances (nerves) [15-17]. Biological time activates (through sensation, digestion, and execution) the space fabric (organs) composed of physical molecules (protein, carbohydrates, etc.). We can easily infer that physical time activates (through receiving, storing, and emitting) the space fabric (primary unit) composed of cosmological substances (energy fields). The unitary logic is applicable to everywhere in the Universe.

In particular, the higher entities are founded on the space fabric of the successive lower world. Intellectual entities are founded on the psychological space fabric, psychological entities are founded on the biological space fabric, biological entities are founded on the physical space fabric, and physical entities are founded on the cosmological space fabric. Each higher entity is found on the lower space fabric using its own time. If the cosmological space fabric is absent, physical time and hence the physical world cannot exist, and all higher entities are voided. For example, when a human biological entity dies, the psychological and intellectual bodies also die. 


\subsection{Difference between the Physical and Cosmological Substances}

Although physical particles are the enablers of biological activation, they follow physical rather than biological laws. Analogously, cosmological particles enable physical activation but follow cosmological rather than physical laws. The author argues that modern physicists have confused physical particles and cosmological particles. We strongly argue that as the space fabric is made of cosmological substances, it cannot be described by physical science. Some important differences between cosmological and physical particles are listed below.

1) A living physical particle "feeds on" (emits and receives) a cosmological substance (heat or charge), but the opposite is not possible.

2) All physical particles such as electrons and protons possess physical mass, physical volume, physical shape, and other physical properties. Conversely, cosmological particles such as photons lack physical mass, physical volume, and physical shape [18]. Photons are treated as both particles and waves with variable wavelengths. Heat can be stored in a physical body of any shape. The theory suggests that any particle possessing physical mass can be categorized as a physical particle; otherwise, it is a cosmological particle. In this view, biological masses (living tissues), psychological masses (emotions), and intellectual masses (knowledge) also lack physical mass and hence do not follow physical laws.

3) Cosmological substances experience no physical distance and no physical time. Cain [19] stated that "there's zero time elapsed between when it's [a photon is] emitted and when it is absorbed again. It [a photon] doesn't experience distance either." Hence, the act of physically moving is meaningless for photons.

4) Quantum theory predicts that "a particle (for instance, a photon) can be at different places at the same time. In fact, it can even be at infinite places at the same time, exactly as a wave" [20]. Therefore, the term "inertial frame of reference" is applicable only to physical entities, not to photons.

5) We can photograph a physical particle, but we cannot photograph cosmological particles such as light, thrust, and gravity. A photon (light particle) performs a photographic act.

6) Bosons and other sub-atomic particles can receive, store, and emit cosmological substances and can hence be cooled to -273.15 [21]. Cosmological substances cannot absorb or emit sub-atomic particles.

7) A cosmological substance is a unit of pure energy [22, 23].

8) In nuclear fusion, we cannot presume the conversion of mass to energy. This paper propounds that a physical mass is composed of cosmological substances, including dark energy. Nuclear fusion destroys physical mass, releasing raw material (cosmological substances). The quantum of cosmological substance remains unchanged during this process.

When discussing the ingredients of space fabric, we should distinguish between physical and cosmological particles. Nafousi described space not as a void but as a fabric made of different cosmological ingredients [5]. The word "vacuum" only describes the non-availability of physical particles, not cosmological particles.

\subsection{Panoramic View of the Universe}

Imagine an ocean filled with various imperceptible cosmological substances, along with cosmological compounds that receive, store, or provide cosmological substances called chests. Under the laws of nature, these compounds are called chests to settle into primary units, where each unit is made of four chests (see section 3 of the paper). Infinitely many of these primary units are united by the self-created binding energy to form the continuous space fabric. The chests within the fabric interact to evolve various kinds of energy fields that accept physical life. This space fabric constitutes our physical Universe. In the panoramic view, our spatial Universe is an island confined within its edges. What lies beyond our island is incomprehensible (see Figure 1). Ellis and Larena [24] suggested that the Universe is curved with closed edges.

In Indian philosophy, space is finite and has a limited scope of activation. All entities are activated over their individual and limited spacetimes (at present, the observable Universe is 93 billion light-years in diameter). Beyond this limit, space fabric exists but is devoid of physical phenomena. A movement of 


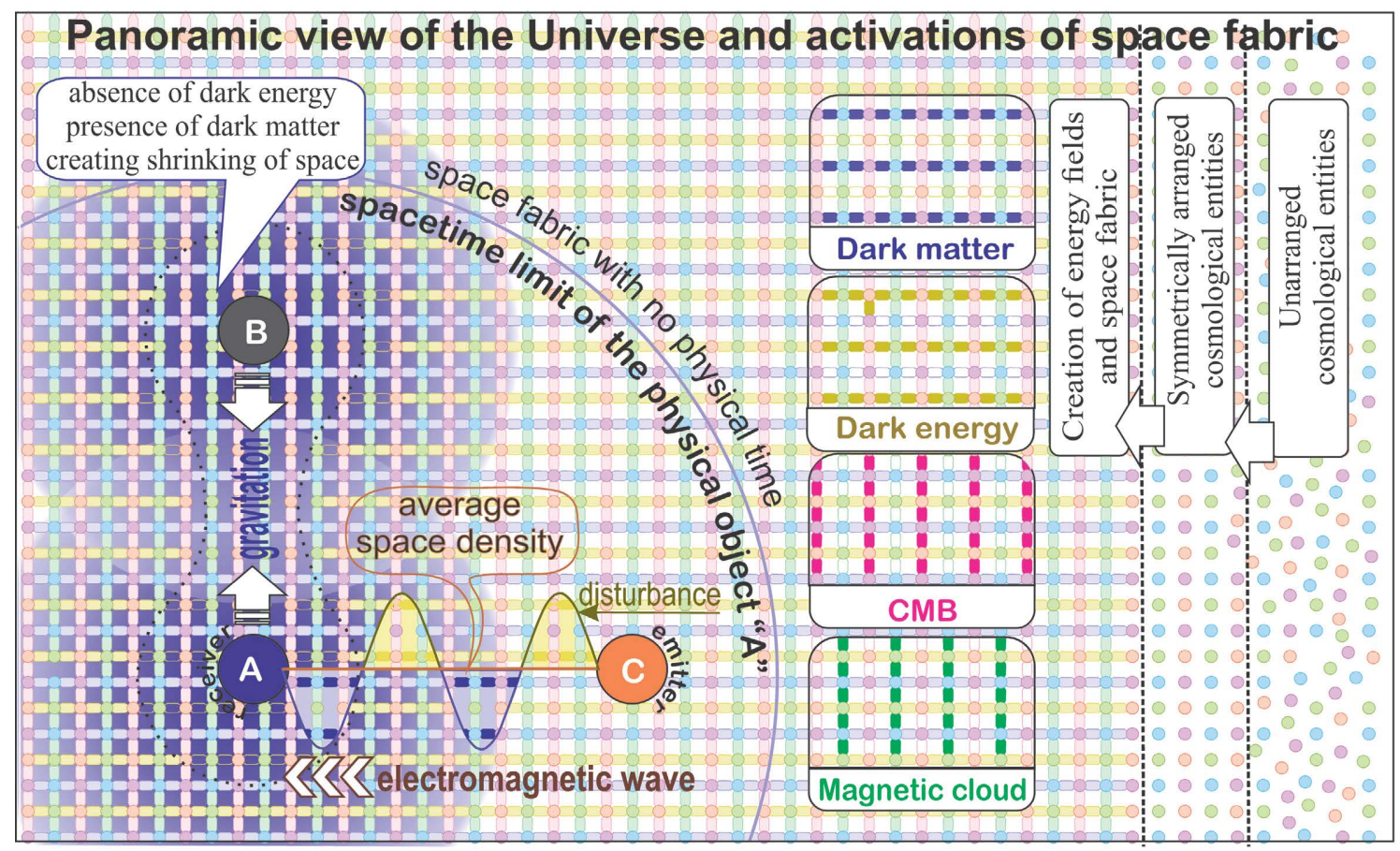

Figure 1. The space fabric of this Universe is composed of symmetrically arranged cosmological substances. The Universe comprises a balanced mix of dark matter, dark energy, visibility, and forcibility, which are responsible for attraction, repulsion, light propagation, and magnetism. In addition, the fabric has flexibility and an average space density that facilitates the creation of waves.

spacetime accompanies a mechanical movement in space. An entity always remains in the center of its spacetime scope, but its movement is restricted to the existing spatial extent.

Above we imagined only one island, but multiple islands or universes may exist in the sea. Two different universes cannot communicate because there is no space fabric between them. The theory suggests that different universes can possess different structures of space fabric, resulting in different types of physical entities. A further discussion is beyond the limits of our intellect.

\subsection{Meaning of Emptiness}

The author posits that emptiness is not filled with the space fabric, but the space fabric itself is the emptiness. The word "emptiness" refers to physical emptiness and not cosmological emptiness. This emptiness allows us to see through because it is made of the space fabric, which allows light waves. Without the space fabric, nothing can be seen. If we remove the space fabric from this emptiness, no emptiness will remain. In particular, the space fabric, aether, and physical emptiness are the same phenomenon. The theory propounds that we have a standard space fabric in the form of emptiness, facilitating a specific speed of light, which depends on the density of the space fabric and is of variable frequencies and amplitudes. The space fabric supports the additional dark energy or dark matter (not a physical matter [25]), resulting in the expansion and shrinkage of the distances. In the case where there is no space fabric or emptiness, the physical world would not exist, thereby resulting in the nonexistence of the biological, psychological, and intellectual worlds.

\subsection{Difference between Space Fabric and Spacetime}

In 1954, Albert Einstein stated that "space and time were merged in a single continuum in a way similar to that in which the three dimensions of space had previously merged into a single continuum. Physi- 
cal space was thus extended to a four-dimensional space which also included the dimension of time." The theory predicts that the (three-dimensional) space fabric (aether) pervades the Universe, and it has a quality to adopt physical time. When a physical entity comprises physical time, the three-dimensional space fabric is converted to a four-dimensional physical spacetime. According to the theory, physical time cannot exist in the absence of space fabric, but space fabric can exist in the absence of physical time. For example, a physical atom can exist without biological life, but biological life cannot exist without a physical atom. A biological life (such as a plant) can exist without psychological life, but a psychological life (an animal) cannot exist without biological life. Ashceñar stated [26] that "In the absence of a spacetime continuum, many of the notions habitually used in physics are no longer available." This statement holds because, in the absence of physical time, space remains as a cosmological fabric that does not obey physical laws.

\section{STRUCTURE OF SPACE FABRIC}

A number of theories have been proposed for the structure of space [27-29], but none of them differentiate between the cosmological fabric and physical time. The present theory propounds that the space fabric is made of cosmological substances, composed of an infinite number of primary units that bond to form the fabric. These primary units exist in the form of small squares, each with four chests that are the basic ingredients of the fabric. All four chests adopt a compromising attitude with opposite behaviors that maintain the fabric in a continuously bonded form. Physical time depends on the physical entity, which over takes the space fabric and uses it as a means for its activities. Please refer to Figure 2.

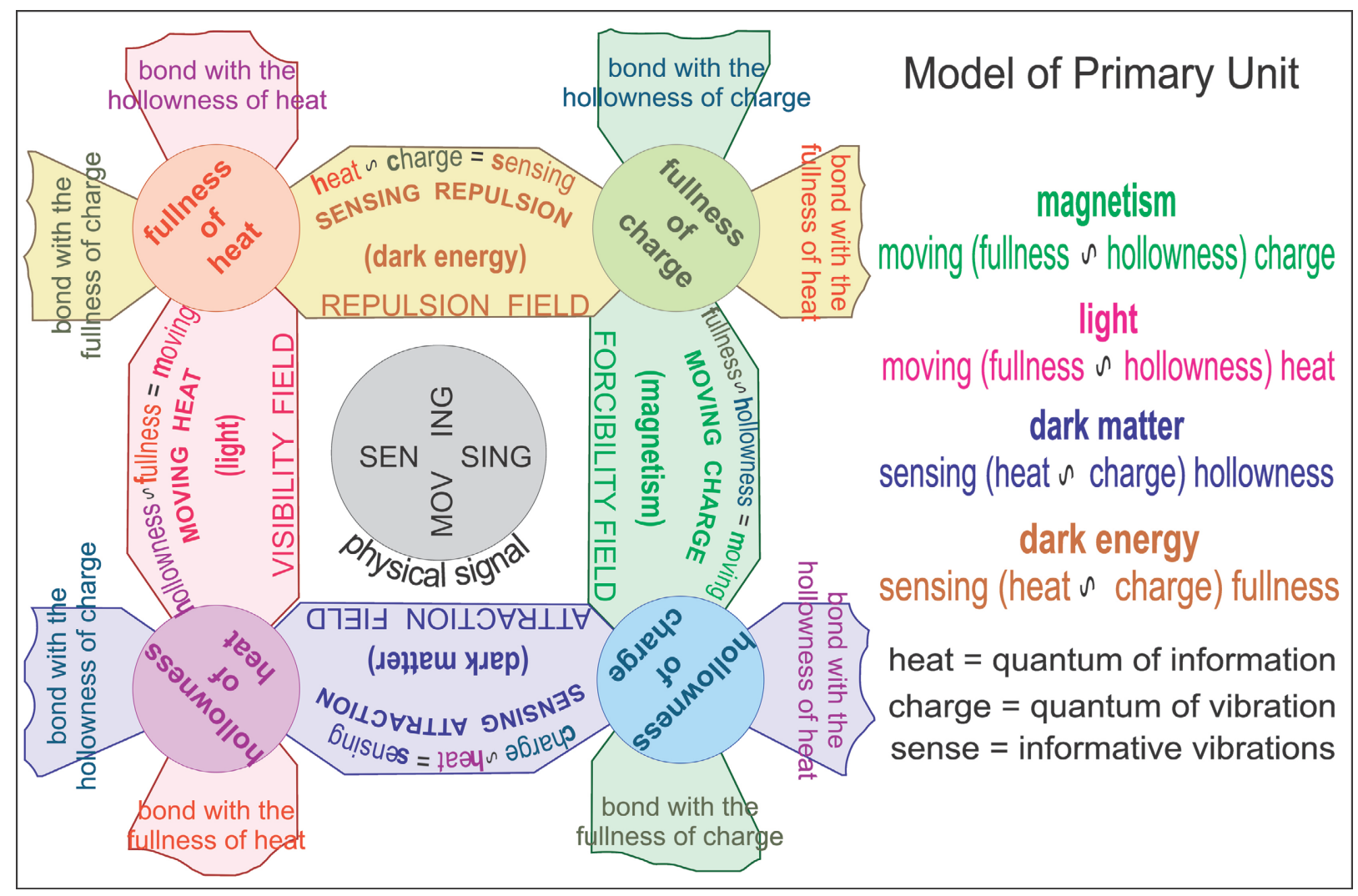

Figure 2. The complete space fabric is composed of small units of four chests. These chests can receive, store, and provide different cosmological substances, creating the four fields: fullness field, hollowness field, forcibility field, and visibility field. These energy fields are the fundamental means of activation. 


\subsection{Essentiality of the Chests}

Entities of all levels have their own storing chests. All entities are capable of receiving, storing, and providing the corresponding data. The intellectual entity stores (memorizes) knowledge. The psychological entity stores emotions. The biological entity stores health. The physical entity stores energy symbols. By applying the unified approach, we can easily infer that a cosmological entity must have some storing procedure (i.e., chests) to store the different aspects of the energy fields in dormant form. Any direct experiment cannot prove the presence of these chests because these chests are imperceptible phenomena. However, their presence is proved by 1) the flexibility of the fabric, which creates waves, gravitation, repulsion, and curvatures, and 2) the evolution of dark energy, which is responsible for the acceleration in the expansion of the Universe; this perceptible evolution of dark energy comes only from the imperceptible chests.

\subsection{Shape of Primary Units (Four Chests)}

Being cosmological substances, we cannot imagine the physical shape of the primary unit. However, for the purpose of understanding, a shape is required. Nature has not provided us the spatial space with four or five dimensions. Hence, we can never think of them. We can only think in three dimensions, which creates a cubic form and covers the complete spatial space without leaving any unused part of it. Without this cubic form, we cannot imagine our space as fully empty. If we accept the cubic formation, we only need four chests to create a complete cube. Please refer to Figure 3.

\subsection{Placement and Number of Chests in a Primary Unit}

There are two parts of the primary unit. 1) The chests, which are a permanent part of the unit, remain imperceptible all the time, and 2) the energy fields, which are a temporary part of the unit, remain perceptible with variable quanta. The chests are the cause, and the fields are the results. Because of the permanency of the cause, the chests are placed at all the corners of the cubes. The chests are placed at the corner of the unit, where it becomes a junction to join eight units with it. The cubic structure can connect in six directions (forward, backward, left, right, up, and down). All chests have their own stored data, and during activation, they transfer to the different primary units. This result makes the activation possible. Please refer to Figure 3.

\subsection{Root Structure of the Fabric (The Four Chests)}

Sophisticated models have proliferated in modern science. Such models seem to contradict the inherent simplicity of nature. When considering the different levels of the Universe, the upper levels are more sophisticated than the lower levels. The cosmological world is the lowest perceptible level and must be the

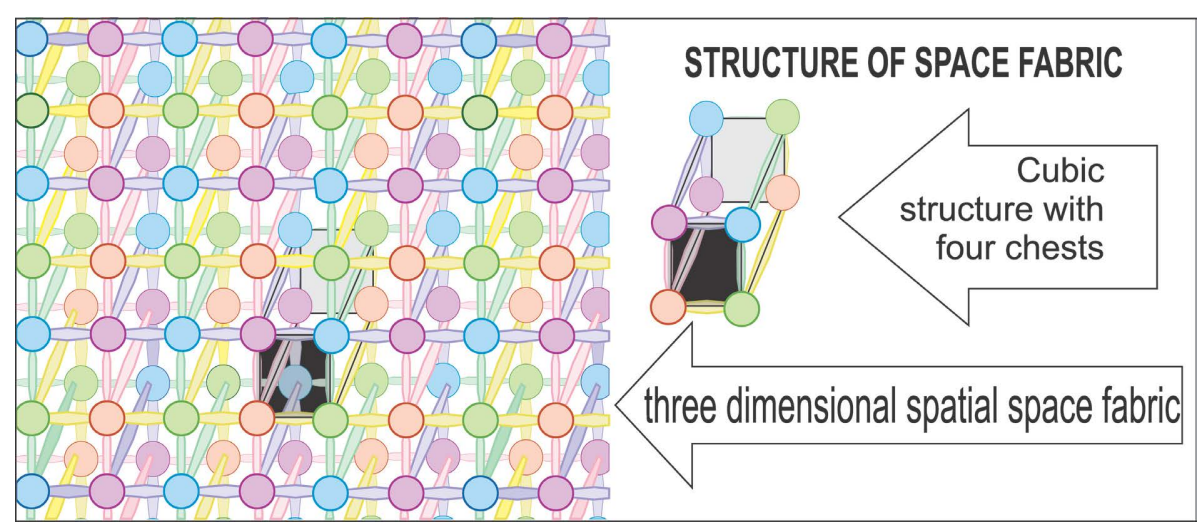

Figure 3. Each primary unit is made of four chests. They are bonded with the opposite phase. In three dimensions, it is cubic in shape, which creates the emptiness in the space. 
simplest form of nature. The chests comprising the primary units are imperceptible. Each chest can receive, store, and provide specific ingredients of different energy fields (see Figure 2). Please note that this model of four chests is philosophical (not mechanical) in meaning. As the chests are imperceptible, they are named according to their nature only: 1) fullness of heat, 2) fullness of charge, 3) hollowness of charge, and 4) hollowness of heat. The names reflect the opposite and supplementary natures of their relationship. Each chest maintains a flexible storage capability, so that they can emit/receive data to/from the feabric. This flexibility is necessary for creating waves. The differences among the chests provoke the following interactions:

1) Fullness of heat: a) When interacting with "hollowness of heat", it creates a moving visibility field (light); b) when interacting with "fullness of charge", it creates a stationary fullness field (dark energy; repulsion).

2) Fullness of charge: a) When interacting with "hollowness of charge", it creates a moving forcibility field (magnetic flux); b) when interacting with "fullness of heat", it creates a stationary fullness field (dark energy; repulsion).

3) Hollowness of charge: a) When interacting with "fullness of charge", it creates moving forcibility (magnetic flux); b) when interacting with "hollowness of heat", it creates a stationary hollowness field (dark matter; attraction).

4) Hollowness of heat: a) When interacting with "fullness of heat", it creates a moving visibility field (light); b) when interacting with "hollowness of charge", it creates a stationary hollowness field (dark matter; attraction).

These interactions lead to four energy fields (visibility, forcibility, hollowness, and fullness fields), as shown in Figure 2.

These fields cannot be treated as fundamental phenomena because 1) these are the effects of the cause coming from the chests, 2) the quanta of these fields are variable (in the case of electromagnetic waves), so their cause is inevitable, and 3) the fields are not independent must be disciplined by some common controller. In our case, the fundamental phenomenon is "chest", which has the flexible storing capability to keep the energy fields in dormant form. In the case of electromagnetic waves, in half of the wave, we need dark energy, and in half of we relieve dark energy and need dark matter. Everything is adjusted within the fabric.

\subsection{Creation of Energy Fields}

The energy fields generated by interactions among the four chests are described below.

1) Visibility field: The interaction between the "fullness of heat" and "hollowness of heat" chests creates a "flow (interaction between fullness and hollowness) of heat" called the visibility field. The excess visibility field appears as cosmic background radiation.

2) Forcibility (magnetic) field: The interaction between the "fullness of charge" and "hollowness of charge" chests creates a "flow (interaction between fullness and hollowness) of charge" called the forcibility field (magnetic flux).

3) Fullness Field: The interaction between the "fullness of heat" and "fullness of charge" chests creates a "sensation (heat + charge) of crowd (fullness)" in the space fabric called the fullness (repulsion) field. The excess repulsion appears as dark energy. Dark energy is responsible for expansion (long distances) of space or reversal of a curve in the space fabric.

4) Hollowness field: The interaction between the "hollowness of heat" and "hollowness of charge" chests creates "sensation (heat + charge) of hollowness" in the space fabric called the hollowness (attraction) field. The excess attraction appears as dark matter. Dark matter is responsible for the shrinkage of the space fabric.

Explanation-Oscillation between "fullness" and "hollowness" carries a wave-like quantum that flows toward a receiver (a lower entropy zone). Heat flow is a quantum of light (information). Analogously, charge flow is a quantum of (vibration). This interaction between "heat" and "charge" creates informative 
vibrations, which is regarded as sensing.

In summary, the fabric is made of symmetrically arranged primary units. Each unit has four chests that create four types of energy fields-dark energy (repulsion), dark matter (attraction), visibility, and forcibility. The visibility is available in the form of background radiation. The interaction between dark matter and dark energy creates a charge. Every charge is exposed to the repulsion and attraction from all sides that balances the system. Nikolskiy stated [30] that "a continuous network made of discrete elementary electric charges that carries the light and also is the source of the background radiation called relict. In the aether network every charge is exposed to the forces of attraction and repulsion front and back, right and left, their action being balanced."

\subsection{Proof That the Fabric Is Made of Energy Fields}

When waves transport moving or inertial signals from one point to another, the ingredients of the signals remain static. In particular, the transported energy fields already exist within the medium of the space fabric. As these four fields play an important physical-activation role in the space fabric, they must be the basic ingredients of the space fabric. Numerous other fields within the space fabric, such as the Higgs field, are not the main players in physical activation. In addition to the above, as we have explained, dark matter and dark energy are just opposite; one is shrinking the space, and the other is expanding the space. Therefore, nature has placed them in a structure so that they cannot wipe off each other and live in a family. The author proposes that the structure shown in Figure 2 is the only possibility of placement. As far as the experimental proof is concerned, everything is out of reach of our physical instrument, not because they are small enough for our observation, but because they are a cosmologically balanced (zero value) phenomenon. Secondly, we cannot find a place with no space fabric; hence, the comparison is impossible.

\subsection{Activation of the Space Fabric}

The space fabric is the medium through which signals are transmitted from one point to another. Transmitting a signal through a wave requires a degree of flexibility. This flexibility is created by the attributes of the four chests (Imperceptible) that receive, store, and provide the visibility, forcibility, attraction, and repulsion fields. As mentioned above, these fields can be quantified in terms of light, magnetism, dark matter, and dark energy. The above four fields are perceptible and take part in the activation. They balance each other and maintain an average space density in the fabric. The space fabric is a physically non-living substance. All physical activations occur when a "time" is introduced by a physical entity. There are two types of time as defined below:

1) Moving time creates light and magnetism: When a disturbance occurs at the emitter end, the flexibility of the fabric creates an oscillation between "fullness" and "hollowness". These oscillations create a wave whose flow is controlled and directed by time. Time always moves toward the receiver, which always lies in the lower entropy zone.

2) Existing time creates attraction and repulsion: Existing time holds physical entities and represents the reference frame of an entity in space. The existing time cannot receive or deliver anything to the entities but senses the changes in the space fabric. The reduction of dark energy or increment of dark matter mainly shrinks the space, resulting in attraction between two points. Oppositely, dark-matter reductions or dark-energy increments create space expansion, resulting in repulsion (antigravity) between two points.

\section{PROOF AND APPLICATION OF THE THEORY IN DIFFERENT COSMOLOGICAL PHENOMENA}

As the cosmological substances do not follow the physical parameters such as mass, volume, shape, placement, and time, we cannot find any direct evidence to support the theory. However, inferences can be drawn from previous experiments, which confirm the correctness of the theory. 
Some of the proven observations that confirm the correctness of the theory are described below:

1) Light: A wave is an oscillation that travels through spacetime, accompanied by a transfer of heat energy. The oscillating fullness (dark energy) and hollowness (dark matter) fields propagate a quantum of heat energy (moving heat) from the emitter to the receiver. Conventionally, this oscillation is called an electric field. If the observer intends to receive radiation, it acquires an active visibility field (without a forcibility field) and evolves a sensation of diversity (information). A propagating heat quantum that carries information is called light. Light is observable (diversified) information.

2) Magnetic flux: Oscillation of the fullness (dark energy) and hollowness (dark matter) fields propagate a quantum of charge energy (moving charge) from the emitter to the receiver. Conventionally, this oscillation is called a magnetic field. If the observer intends to obtain a magnetic flux, it acquires the active forcibility field (without a visibility field) and evolves a sensation of intensity (vibrations). The propagation of a quantum of charge through vibrations is called magnetism. Magnetism is a form of polarized (intensified) vibration.

3) Gravitation: Interaction between the visibility and forcibility fields evokes a sensation between two distinct bodies. The hollowness field (without a fullness field) between the bodies evolves a pulling action. That is, attraction evolves through the stationary sensation of pulling.

4) Dark matter: The Swiss astronomer Fritz Zwicky noticed that some distant galaxies were orbiting other galaxies at much faster speeds than expected from their visible masses [31]. He proposed the existence of an unseen mass called dark matter. This mysterious mass has been confirmed throughout the cosmos. In the present theory, this phenomenon describes the "hollowness field" created by excess "hollowness of heat" and "hollowness of charge" within the space fabric. The hollowness field shrinks the space fabric and creates gravitational attraction, which is sensed by the "existing time" of the nearby massive bodies.

5) Dark energy: The theory proposes that dark energy is the "fullness field" (crowd) created by excess "fullness of heat" and "fullness of charge" within the space fabric. The fullness field expands the space fabric and creates a repulsive effect that is sensed by the "existing time" of the nearby massive bodies.

6) Cosmic repulsive force: The oscillation of the visibility and forcibility fields evokes a sensation between two distinct bodies. The fullness field (without a hollowness field) between the bodies evolves a repulsive effect. That is, repulsion evolves through the stationary sensation of pushing. Dark energy is known to create repulsive forces [32], as can be observed in the ejection of plasma from the Sun's corona and the direction of the tail of a comet near the Sun.

7) Cosmic microwave background ( $\mathrm{CMB}$ ): The theory proposes that the CMB is a "visibility field" caused by excess "fullness of heat" and "emptiness of heat" within the space fabric. The visibility field illuminates space and provides light quanta to an observer. Because both chests responsible for the visibility field are imperceptible and no emitter is available for radiation, the available illumination apparently comes from the background of the space fabric (i.e., the CMB). Nikolskiy stated [30] that "a continuous network made of discrete elementary electric charges that carries the light and also is the source of the background radiation called relict."

8) Diversion of light by massive physical bodies: A massive physical body in the space fabric continuously consumes a cosmological diet (dark energy in our case), creating a local zone of imbalanced dark matter. This dark matter shrinks the space, increasing its curvature. When light from a distinct star crosses the nearby space fabric, it is diverted toward the hollowness (dark matter) in the space fabric. This phenomenon appears as a diversion of light toward a massive star.

9) Expansion of the Universe: Modern cosmology has been largely inspired by the current discovery of the accelerated expansion of the Universe [33-36]. This unexpected acceleration has been explained by introducing dark energy into General Relativity. The present theory suggests that dark energy evolves through interactions between "fullness of heat" and "fullness of charge", which create a strong antigravity effect. When describing an expanding Universe, we refer not to the size of the Universe but to the distances between objects in the Universe. Here, "distance" has a specific meaning, as explained below. 
10) Distances in the space fabric: In space, physical distance measurements give the length of the pathway across space. Imagine crossing a sea in a motorboat operated by a jet engine. The speed of the motorboat will depend on the density of the water, which is constant. Hence, we will obtain the same result whether we measure the distance in kilometers or in time. If the density of water is variable, the time and distance measurements will differ. Therefore, the distance should be measured in terms of time only, although the sea passage can be measured in terms of kilometers. An analogous situation occurs for electromagnetic waves flowing through the space fabric. If the space-fabric density is homogenous, the travel time between two points separated by the same distance will be constant, but if the space-fabric density is variable or curved (due to dark energy or dark matter), the travel time can vary. In terms of time, the distance between two points in the space fabric depends on the spacetime curvature [37].

11) Speed of light: The speed of light depends on the medium through which the light propagates (i.e., the space fabric or aether). As this medium homogeneously pervades the Universe, the speed of light is constant through space. When there is excess dark energy or dark matter, the speed can increase or decrease, but assuming constant speed, we can consider the differences in distances. In regions of excess dark energy and dark matter, the distance can increase (cosmic inflation) or decrease (gravitational lensing of light), respectively.

12) Cosmological constant problem: In dense space, the theoretical data largely deviate from the observational data. Our space fabric is composed of four energy fields that oppose each other. Within this space fabric, we observe only the resultant, which is nearly 0 .

\section{CONCLUSIONS}

Many mysteries in quantum physics remain to be solved. The author suggests that to solve these mysteries, we must identify their root cause. The concept of space fabric is still developing and must be elucidated to understand quantum physics at its most fundamental level. The main conclusions of this paper are summarized below.

1) A cosmological substance can never be physically alive. Hence cosmological photons and physical electrons should be treated in different paradigms.

2) The space fabric (or aether) is a cosmologically knotted fabric that mediates the activation of physical entities.

3) Dark matter is not physical matter but a tendency of the space fabric.

4) We cannot state that something fills the emptiness of space to create the space fabric. Instead, the space fabric creates the emptiness of space.

5) Time is a physical element that evolves via the entropy difference between two physical objects. It can be defined as a "willingness to activate".

6) When physical time activates, it immediately acquires cosmological space fabric, creating a four-dimensional spacetime continuum.

7) The space fabric can exist without time, but time cannot exist without the space fabric. Analogously, we propose that physical matter can exist without biological life but that biological life cannot exist without physical matter.

8) A model of the space fabric is suggested, where it is made up of an infinite number of interlinked primary units. These primary units are made of four imperceptible chests: fullness of heat, fullness of charge, hollowness of heat, and hollowness of charge. These four imperceptible chests evoke the four perceptible energy fields: the visibility, forcibility, attraction, and repulsion fields. These fields facilitate the activation of light, magnetism, gravity, and repulsion.

9) The model explains mysterious phenomena such as dark matter, dark energy, cosmic inflation, diversion of light by gravity, and the $\mathrm{CMB}$, etc.

The basic limitation with the paper is that it has used the facts accepted by the various science scholars, and no new experiment is proposed. We know that cosmology is the subject where physical observations can help only a bit and that work has already been done. Further, our subject is more elementary, 
related to pure cosmology. Here, no reach of any experiment is possible.

This paper presents a new interpretation of so-called space that resolves several mysteries of the Universe. The correctness of the approach is validated by its consistency with the results of well-known experiments. At present, our understanding of cosmology is rudimentary. We consider that once the detailed structure of the space fabric is understood, the cosmological world will be much simplified and that its mysteries will be unveiled. Ultimately, we might realize Einstein's dream of a grand unified theory because the space fabric is the common activation source of both electromagnetic waves and gravity.

\section{ACKNOWLEDGEMENTS}

I would like to thank Prof. Dayanand Bhargava for his suggestions in the field of philosophy. Further, I wish to extend my special thanks to Dr. Ravi Sharma (Apollo Achievement Award, NASA) and Dr. K R Soni (HOD Physics, M N I T, Jaipur) for their important suggestions in the field of science. The author declares that he has no conflict of interest with anyone.

\section{CONFLICTS OF INTEREST}

The author declares no conflicts of interest regarding the publication of this paper.

\section{REFERENCES}

1. Einstein, A. (1920) Ether and the Theory of Relativity. Methuen, London, 1922. https://doi.org/10.1007/978-94-011-6022-3_2

2. Hinckfuss, I. (1995) Review of What Spacetime Explains; the Shape of Space, by G. Nerlich. British Journal for the Philosophy of Science, 46, 425-435. http://www.jstor.org/stable/687667 https://doi.org/10.1093/bjps/46.3.425

3. Wise Thomson, D. and Bourassa, J.D. (2004) Secrets of the Aether. Quantum Aether Dynamics Institute. https://doi.org/10.13140/2.1.1633.7926

4. Forrest, P. (2013) The Necessary Structure of the All-Pervading Aether. De Gruyter, Berlin. https://doi.org/10.1515/9783110325928

5. Nafousi, M. (2019) The Fabric of Space-Nature and Structure. The Binary Universe (Book). https://medium.com/@MahmoudNafousi/the-pixelated-fabric-of-space-5ea178694caf\#: :text=The\%20vacuum \%20exhibits\%20zero\%20charge,is\%20a\%20foam\%2Dlike\%20structure

6. Forrest, P. (2013) The Necessary Structure of the All-Pervading Aether, Discrete Or Continuous? Simple or Symmetric? Philosophical Analysis, Vol. 49, De Gruyter, Berlin. https://doi.org/10.1515/9783110325928

7. Agrawal, P.K. (2021) An Alternative Approach toward the Origin of the Universe. Philosophy and Cosmology, 27, 5-21. https://doi.org/10.29202/phil-cosm/27/1

8. Radhakrishanan, S. (1968) The Principle Upanishads. George Allen \& Company Ltd., p. 546. https://ia802507.us.archive.org/10/items/PrincipalUpanishads/129481965-The-Principal-Upanishads-by-S-Rad hakrishnan.pdf

9. Haldane, J., Thompson, D., Mitchell, P. and Hobhouse, L. (1918) Symposium: Are Physical, Biological and Psychological Categories Irreducible? Proceedings of the Aristotelian Society, Supplementary Volumes, Vol. 1, 11-74. http://www.jstor.org/stable/4106485 https://doi.org/10.1093/aristoteliansupp/1.1.11

10. Camp, J. (2012) Decisions Are Largely Emotional, Not Logical. https://bigthink.com/experts-corner/decisions-are-emotional-not-logical-the-neuroscience-behind-decision-ma king 
11. Jung, N., Wranke, C., Hamburger, K. and Knauff, M. (2014) How Emotions Affect Logical Reasoning: Evidence from Experiments with Mood-Manipulated Participants, Spider Phobics, and People with Exam Anxiety. Frontiers in Psychology, 5, 570. https://doi.org/10.3389/fpsyg.2014.00570

12. Izard, C.E. (2009) Emotion Theory and Research: Highlights, Unanswered Questions, and Emerging Issues. Annual Review of Psychology, 60, 1-25. https://doi.org/10.1146/annurev.psych.60.110707.163539

13. Gobar, A. (1968) Psychology of Thought Processes. In: Philosophic Foundations of Genetic Psychology and Gestalt Psychology, Springer, Berlin, 117-165. https://doi.org/10.1007/978-94-015-0813-1_5

14. Sternberg, R.J., Berlyne, D.E. and Vinacke, W. (2008, May 14) Thought. https://www.britannica.com/topic/thought

15. McLeod, S. (2015) Psychology Perspectives. https://www.simplypsychology.org/perspective.html

16. SparkNotes Editors (2005) SparkNote Page 1. The Biological Bases of Emotion. Emotion. https://www.sparknotes.com/psychology/psych101/emotion/section2/

17. Johnson, J.A. (2016) Biology Determines Every Thought, Feeling, and Behavior. Psychology Today. https://www.psychologytoday.com/us/blog/cui-bono/201610/biology-determines-every-thought-feeling-and-be havior

18. Vasilev, G.S., Ljunggren, D. and Kuhn, A. (2010) Single Photons Made-to-Measure. New Journal of Physics, 12, Article ID: 063024. https://doi.org/10.1088/1367-2630/12/6/063024

19. Cain, F. (2014, May 7) Does Light Experience Time? Universe Today; Space and Astronomy News. https://www.universetoday.com/111603/does-light-experience-time

20. University of Bristol (2012) “One Real Mystery of Quantum Mechanics": Physicists Devise New Experiment. https://phys.org/news/2012-11-real-mystery-quantum-mechanics-physicists.html

21. Perkowitz, S. (2008) Bose-Einstein Condensate. Encyclopaedia Britannica. https://www.britannica.com/science/Bose-Einstein-condensate

22. Madeleine, O. (2019) Massless Particles Can’t Be Stopped. https://www.symmetrymagazine.org/article/massless-particles-cant-be-stopped

23. Rugh, S.E. and Zinkernagel, H. (2002) The Quantum Vacuum and the Cosmological Constant Problem. Studies in History and Philosophy of Science Part B, 33, 663-705. https://doi.org/10.1016/S1355-2198(02)00033-3

24. Ellis, G. and Larena, J. (2020) The Case for a Closed Universe. Astronomy and Geophysics, 61, 1.38-1.40. https://doi.org/10.1093/astrogeo/ataa011

25. Siegel, E. (2020, March 13) 5 Things We Know about Dark Matter (And 5 We Don't). Forbes. https://www.forbes.com/sites/startswithabang/2020/03/13/5-thing-we-know-about-dark-matter-and-5-we-dont /? sh=5837d 26036f8

26. Ashceñar, A. (2006) Space and Time: From Antiquity to Einstein and Beyond. Resonance, 16 and 18. https://www.ias.ac.in/article/fulltext/reso/011/09/0004-0019

27. Krasnoholovets, V. (2017) Structure of Space and the Submicroscopic Deterministic Concept of Physics. Apple Academic Press.

https://www.routledge.com/Structure-of-Space-and-the-Submicroscopic-Deterministic-Concept-of-Physics/Kra snoholovets/p/book/9781774636718

28. Singh, T.P. (2018) Quantum Theory and the Structure of Space-Time. Zeitschrift für Naturforschung A, 73, 733-739. https://doi.org/10.1515/zna-2018-0259

29. Hawking, S. and Ellis, G. (1973) The Large Scale Structure of Space-Time (Cambridge Monographs on Mathematical Physics). Cambridge University Press, Cambridge. 
30. Nikolskiy, G. (2018) The Charge Structures of Aether and Substance. Open Access Library Journal, 5, 1-9. https://www.scirp.org/journal/paperinformation.aspx?paperid=82478 https://doi.org/10.4236/oalib.1104379

31. Winkler, K. (2014) Fritz Zwicky and the Search for Dark Matter. Swiss American Historical Society Review, 50, 23-41. https://scholarsarchive.byu.edu/facpub/1991

32. Barghout, K. (2019) Analysis of Repulsive Central Universal Force Field on Solar and Galactic Dynamics. Open Physics, 17, 364-372. https://doi.org/10.1515/phys-2019-0041

33. Mishra, B., Ray, P.P. and Pacif, S.K.J. (2018) Anisotropic Cosmological Models with Two Fluids. Advances in High Energy Physics, 2018, Article ID: 6306848. https://doi.org/10.1155/2018/6306848

34. Kastner Ruth, E. and Stuart, K. (2018) Are Dark Energy and Dark Matter Different Aspects of The Same Physical Process? Frontiers in Physics, 6, 71. https://www.frontiersin.org/article/10.3389/fphy.2018.00071 https://doi.org/10.3389/fphy.2018.00071

35. Ruth, D. (2011) What Do We Really Know about Dark Energy? Philosophical Transactions of the Royal Society of London Series. Part A, 369, 5102-5114.

36. Ball, P. (2003, July 23) Direct Evidence Found for Dark Energy.

37. Solà, J. (2015) Vacuum Energy and Cosmological Evolution. AIP Conference Proceedings, 1606, 19. https://doi.org/10.1063/1.4891113 